\title{
Preoperative Incidence of Femoral Deep Vein Thrombosis after proximal femur fracture at Broomfield Hospital
}

\author{
Hamad Latif FRCA, EDRA, Broomfield Hospital \\ Russel Emamdee FRCA, Broomfield Hospital \\ Email: hamad.latif@meht.nhs.uk
}

\section{Introduction}

Deep vein thrombosis (DVT) is a known cause of morbidity in elderly patients requiring proximal femur fracture surgery

These patients exhibit all risk factors of Virchow's triad: immobilization; advanced age; comorbidity. Surgery later than 24 hours post-injury increases mortality secondary to DVT complications

Current guidelines recommend mechanical and pharmaceutical prophylaxis; however, perioperative DVT is still known to occur in $9-62 \%$ of patients receiving prophylaxis

The purpose of this study is to determine the preoperative incidence of DVTs in our cohort of proximal femur fracture patients

\section{Methods \& Materials}

After audit committee approval, consecutive patients $>60$ years on the principal investigators' proximal femur fixation list were studied. Ultrasound scan was done bilaterally, prior to (unilateral) Fascia liaca block (FIB)

Data collected included demographics, comorbidities, and current DVT prophylaxis

The scan determined the compressibility and flow in the femoral veins (fully compressible, good flow: no DVT; not fully compressible, limited flow, with crescent-shaped clot: suggestion of DVT)

\section{Results}

From January to April 2019, 27 patients were studied

Average age 82 years ( 63-100).

18 Female, 9 male

The mean time to procedure post-injury was 31 hours (13.5-50).

Almost half of patients received interim DVT prophylaxis

Two (7.4\%) patients had a positive result for a DVT

However, no patient had pre-existing signs or symptoms suggesting DVTs

No patient developed pulmonary embolism 24 hours postoperatively

\section{Conclusions}

The incidence of DVT after proximal femur fracture is relatively low at Broomfield Hospital

We surmise that a simple ultrasound observation of the femoral vessels at FIB will determine the true prevalence of DVTs in this cohort of patients 